\title{
A surveillance method-oriented detection of post-operative spatial-temporal recurrence for non-small cell lung cancer
}

\author{
Jun-Jie Xi", Jia-Cheng Yin", Lin Wang, Chun-Lai Lu, Qun Wang, Wei Jiang \\ Department of Thoracic Surgery, Zhongshan Hospital of Fudan University, Shanghai 200032, China \\ Contributions: (I) Conception and design: JC Yin, JJ Xi, W Jiang, Q Wang; (I) Administrative support: W Jiang, Q Wang; (I) Provision of study \\ materials or patients: L Wang, JC Yin, CL Lu; (I) Collection and assembly of data: JC Yin, JJ Xi, W Jiang, L Wang; (I) Data analysis and \\ interpretation: JC Yin, JJ Xi, CL Lu; (I) Manuscript writing: All authors; (I) Final approval of manuscript: All authors. \\ \#These authors contributed equally to this work. \\ Correspondence to: Dr. Wei Jiang, MD; Dr. Qun Wang, MD. Department of Thoracic Surgery, Zhongshan Hospital of Fudan University, No. 180, \\ Fenglin Road, Shanghai 200032, China. Email: jw_zshospital@163.com; wang.qun@zs-hospital.sh.cn.
}

Background: This study evaluated spatial-temporal recurrence patterns after curative resection for nonsmall cell lung cancer (NSCLC) to clarify and recommend appropriate post-operative surveillance.

Methods: A total of 2,486 consecutive patients between January 2005 and December 2012 with NSCLC (stage I-IIIA) who underwent definitive surgical resection were retrospectively analyzed. We used a hazard rate curve to evaluate event dynamics. Disease-free survival (DFS) was evaluated by the Kaplan-Meier method. Univariate and multivariate analyses with Cox proportional hazards regression identified risk factors that predicted DFS.

Results: The median follow-up was 50.1 months. Recurrences were diagnosed in 852 (34.3\%) patients. Four hundred eighty-nine events first occurred in the chest, 177 in the brain, 117 in the bone, and 71 in the abdomen. Of all recurrences, $78.5 \%$ occurred in the first 3 years. Univariate and multivariate analyses identified the age at diagnosis $(\mathrm{P}<0.001)$, histology $(\mathrm{P}=0.023)$, tumor size $(\mathrm{P}<0.001)$, pathologic $\mathrm{N}$ stage $(\mathrm{P}<0.001)$, and grade $(\mathrm{P}=0.043)$ as independent risk factors for intra-thoracic recurrences. Histology $(\mathrm{P}<0.001)$, tumor size $(\mathrm{P}<0.001)$, surgical method $(\mathrm{P}=0.021)$, pathologic $\mathrm{N}$ stage $(\mathrm{P}<0.001)$, and grade $(\mathrm{P}=0.005)$ were independent to predict extra-thoracic recurrences. The hazard rate curve displayed an initial surge of time to any treatment failure during 12 months after surgery. Based on sub-group analysis, both intra- and extra-recurrences increased with stage and brain recurrences in stage IIIA occurred earlier than stage II. Hazard rate curve of brain recurrences in squamous cell carcinoma showed a moderate peak during 9-15 months. Hazard rate curves of brain and bone recurrences in adenocarcinoma displayed clear peaks at 9-27 and 15-30 months, respectively.

Conclusions: Intra- and extra-thoracic recurrences correlate with different clinicopathological factors. Brain MRI and bone ECT were recommended for selected patients in particular time to early detect extrathoracic recurrences.

Keywords: Non-small cell lung cancer (NSCLC); surveillance; post-operative; recurrence dynamic

Submitted May 07, 2018. Accepted for publication Sep 06, 2018.

doi: $10.21037 /$ jtd.2018.10.32

View this article at: http://dx.doi.org/10.21037/jtd.2018.10.32

\section{Introduction}

Lung cancer (LC) is the most common cause of cancerrelated deaths among malignant tumors worldwide (1). Nonsmall cell lung cancer (NSCLC) accounts for approximately
$80 \%$ of all LC cases. In spite of the adoption of computed tomography screening and advances in surgery, patients undergoing complete resection remain at high risk for local or distant recurrences, even in early stages. The estimated 
5 -year survival rate after surgical resection is only $41 \%$ for stage IIIA NSCLC (2). Thus, recurrence plays an important, but negative role in long-term survival. In fact, the incidence of recurrences in surgically resected NSCLC has been well-described. Kelsey et al. (3) reported that the 5 -year actuarial risk of any recurrences after surgery for early-stage $\mathrm{LC}$ is $36 \%$.

Patients with NSCLC exhibit wide variations in the postoperative recurrence pattern. The distribution of recurrence in different sites has not been well-described, including the incidence over time. There appears to be few general rules in guidelines about how to monitor post-operative patients. Previous studies have reported no benefit in early detection of recurrences to overall survival in those years without effective targeted drugs $(4,5)$. It is worth mentioning that targeted drugs, such as gefitinib and erlotinib, are alternatives presenting promising efficacy in select recurrence cases. Recent findings from the global phase III ALEX trial showed that alectinib appears to avert the progression of CNS metastases in ALK-positive NSCLC patients and baseline CNS metastases, and to reduce the incidence of new CNS metastases (6). Such progress implies the necessity to re-evaluate early detection of recurrences and estimate post-operative recurrence patterns to guide surgical or adjuvant therapies.

In this study we aimed to explore the dynamics of recurrence rates of different sites to determine whether or not and when intense surveillance is needed for post-operative patients and to propose the appropriate follow-up pattern.

\section{Methods}

\section{Data source}

We recruited study participants treated at Shanghai Zhongshan Hospital Thoracic Surgery Department in accordance with a protocol approved by the Institutional Review Board of Shanghai Fudan University [a total of 2,486 consecutive patients (1,586 males and 900 females)] with NSCLC from stage I-IIIA who underwent definitive surgical resection between January 2005 and December 2012. The medical records were retrospectively reviewed to describe the individual demographic, surgical, and pathologic information, and estimate local or distant recurrences.

\section{Study cohort}

We limited the analysis to patients with stage I-IIIA disease based on the eighth edition of the tumor-node-metastasis (TNM) staging by the International Association for the Study of Lung Cancer (IASLC) (2), who had undergone surgical resection, and with at least 12 months of continuous enrollment. Only patients with complete resection were included in the analysis. Anatomic pulmonary resection was performed for the majority of patients. For patients underwent sublobar resection, including segmentectomy and wedge resection, complete resection was achieved by guaranteeing that parenchymal resection margins were not less than $2 \mathrm{~cm}$ or the size of the nodule from primary lesion. Mediastinal lymph node dissection or sampling was performed on all patients. Histologic sub-types were according to the World Health Organization classification. Exclusion criteria included the following: (I) diagnosed at the time of death or died within 30 days after surgery; (II) history of a malignancy; (III) resection performed outside Zhongshan Hospital or incomplete resection, including positive margins; (IV) missing recurrence or survival records; and (V) post-operative mortality without a recurrence.

\section{Statistical methods}

The post-operative visit was provided with the treating surgeon, and follow-up regimen is shown in Table 1 . The primary endpoint of our study was spatial-temporal recurrence patterns. Disease-free survival (DFS), referring to the period after curative resection when no disease could be detected, was recorded and analyzed. Recurrences evaluated in our study included any local recurrence and distant metastasis. Twenty-seven patients developed recurrences in more than one region when recurrences were detected for the first time and were excluded because it was unclear whether or not recurrences occurred at the same time or in sequence. Second primary LCs were excluded. The evaluated sites include lung, brain, liver, bone, adrenal gland, pleura, pericardium, spleen, and regional lymph nodes, which were categorized into intra- and extra-thoracic sites (brain, bone, and abdomen). Time-to-treatment failure was defined as the interval between the date of surgery and the date of recurrence, and patients who were still alive were censored at the date of last follow-up. Death before recurrence events were excluded, and only the first event was considered.

Cumulative recurrence-free curves were constructed using the Kaplan-Meier method and were compared using the log-rank test. Patient variables with a predictive value 
Table 1 Follow-up regimen of the study population

\begin{tabular}{lll}
\hline Surveillance period & Frequency & Monitoring items \\
\hline $1^{\text {st }}$ year & Every 3 months & H\&P, non-contrast-enhanced chest CT and color ultrasound of the abdomen and neck \\
$2^{\text {nd }}$ year & Every 6 months & Vide supra \\
$3^{\text {rd }}$ year and later & Annually & Vide supra \\
\hline
\end{tabular}

Note: residual or new radiographic abnormalities may require more frequent imaging; PET/CT or brain MRI is not routinely indicated, and was performed if there was symptomatic suspicion of a recurrence. H\&P, history and physical examination; PET/CT, positron emission tomography/computed tomography; MRI, magnetic resonance imaging.

of recurrence were identified by Cox proportional hazards regression and reported as hazard ratios (HRs). Variables with $\mathrm{P}$ values $<0.1$ on univariate analysis (UVA) were included in multivariate analysis (MVA).

Compared with the median progression-free survival and cumulative incidence curves, the event-specific hazard rate provides information about incidences over time and was applied to estimate the post-operative recurrence patterns visually. First, patients at risk and events that occurred during a defined time interval ( 3 months in this study) were counted respectively. Second, the life-table method was applied to estimate the hazard rate every 3 months. The hazard rate was measured as the "number of events/ patients at risk per 3-month interval." Third, a scatter plot was made according to the hazard rate changes over time. Considering the random variation of hazard rates which would cause the instability, a LOWESS line smoothing was adopted to smooth the curve and make the consequences closer to reality. The hazard rate curve was truncated when the number of patients at risk was $<100$.

Statistical analysis was performed using IBM SPSS statistics (version 22.0) and $\mathrm{R}$ (version 3.4.3) software (http://www.r-project.org/). The R package included survival, rms, and ggplot2. Statistical significance was set at a two-sided $\mathrm{P}$ value $<0.05$.

\section{Results}

\section{Patients characteristics}

Two thousand four hundred and eighty-six patients were identified in the analysis. The characteristics of this population are listed in Table 2. The maximum follow-up was 112 months and the median follow-up was 50.1 months. Disease recurrence developed in $34.3 \%(852 / 2,486)$ patients. There were 489 events first occurring in the chest, 177 events in the brain, 117 events in the bone, and 71 events in the abdomen. Of the recurrences, 21.3-28.8\% occurred each year after resection for the first 3 years and accounted for $78.5 \%$ of all recurrences. The 3 -year DFS rates were $85.6 \%$ (95\% CI: 83.6-87.6\%), 62.6\% (95\% CI: 58.6-66.6\%), and 44.7\% (95\% CI: 40.4-49.0\%) for patients with stage I, stage II, and stage IIIA disease, respectively. Recurrence developed throughout 6 years in stage I disease with a flat decrease of the DFS rate after 3 years. The recurrence-free probability curves of p-stage I, II, and IIIA are shown in Figure 1. The 5-year DFS of patients in our study was $63.7 \%$ (95\% CI: 61.7-65.7\%).

\section{Univariate and MVA for intra- and extra-thoracic recurrences}

MVA included all covariates with a $\mathrm{P}$ value $<0.1$ in UVA. As the AJCC stage was correlated with the TNM stage, the AJCC stage was not included in the MVA to avoid collinearity among factors. Surgical type includes lobectomy/bilobectomy, sublobectomy and pneumonectomy. Surgical method includes video-assisted thoracoscope surgery (VATS), open surgery and hybrid VATS. Results of the UVA and MVA Cox proportional hazards regression are shown in Table 3. MVA found that age at diagnosis $(\mathrm{P}<0.001)$, histology $(\mathrm{P}=0.023)$, tumor size $(\mathrm{P}<0.001)$, pathologic $\mathrm{N}$ stage $(\mathrm{P}<0.001)$, and grade $(\mathrm{P}=0.043)$ independently predicted intra-thoracic recurrences. With respect to extra-thoracic recurrences, histology $(\mathrm{P}<0.001)$, tumor size $(\mathrm{P}<0.001)$, surgical method $(\mathrm{P}=0.021)$, pathologic $\mathrm{N}$ stage $(\mathrm{P}<0.001)$, and grade $(\mathrm{P}=0.005)$ were independent predictors.

\section{Hazard rate curves of spatial-temporal recurrences}

The hazard rate curve was first analyzed for intra- and extra-thoracic recurrences in all 2,486 patients. The resulting curve (Figure 2) showed a post-operative platform 
Table 2 Patient Characteristics of the study population

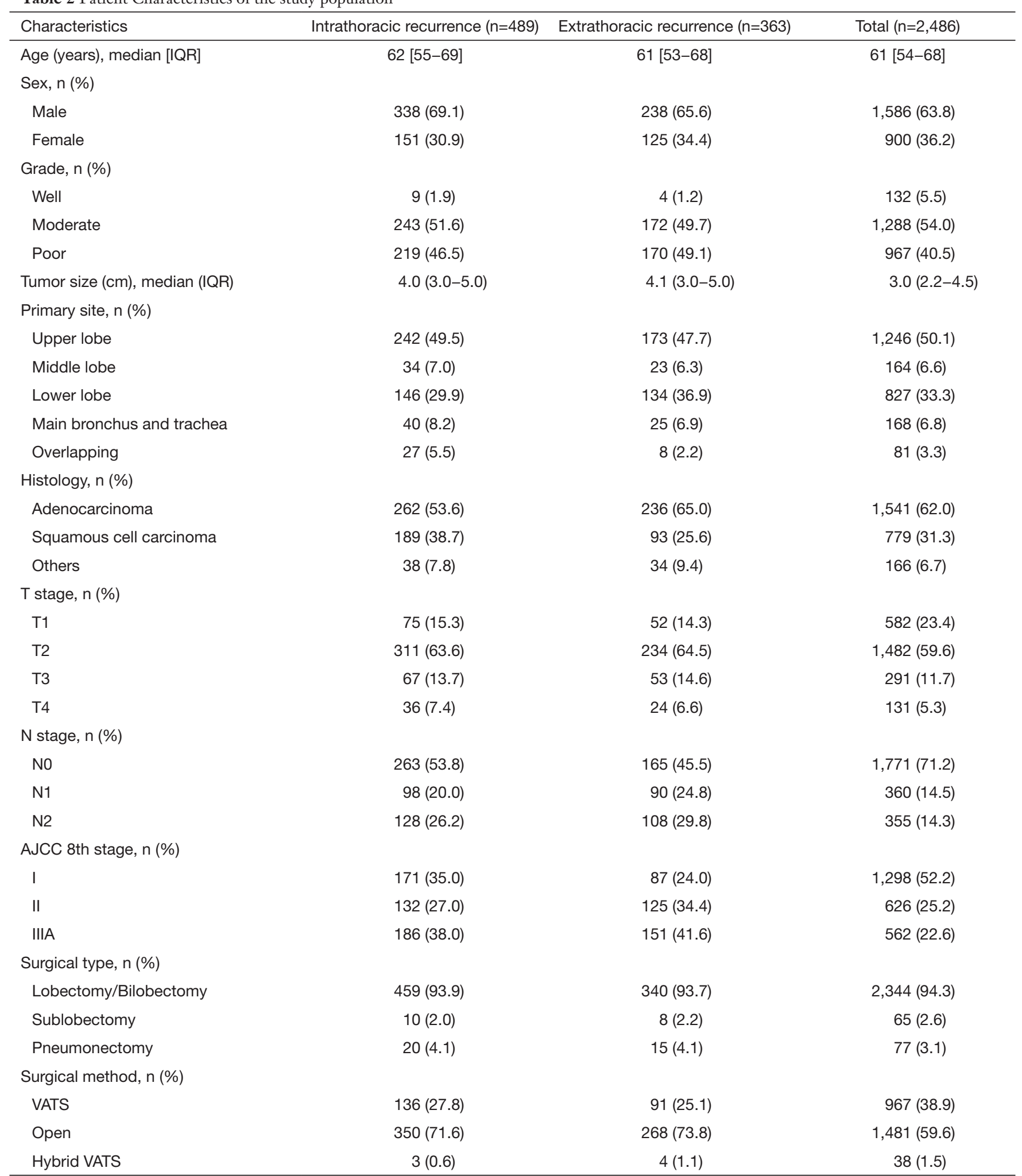

IQR, interquartile range; VATS, video-assisted thoracoscopic surgery. 


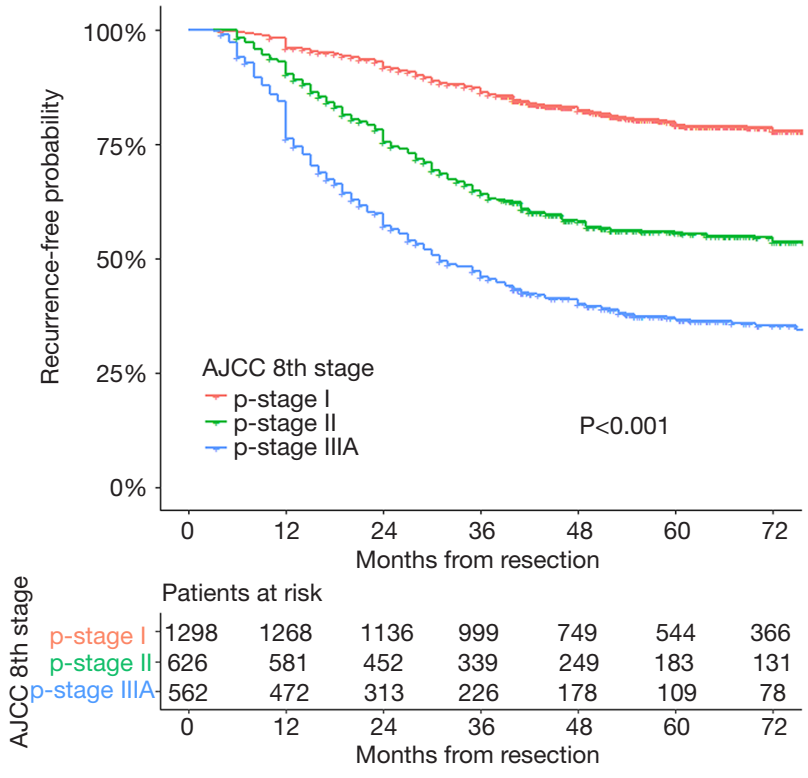

Figure 1 Recurrence-free probability curves of pathological stage I, II and IIIA.

with a high hazard rate $(\mathrm{HR}>0.15)$ at $9-30$ months for intra-thoracic recurrences, which peaked around 12 months (HR $=0.17)$. With respect to extra-thoracic recurrences, an initial surge was displayed in the hazard rate curve (HR $=0.15)$ at $12-15$ months. The hazard rate of intraand extra-thoracic recurrences decreased gradually after 30 months and 12 months, respectively.

Hazard rate curves were then depicted according to pathological stages. In stage I (Figure $3 A$ ), intrathoracic recurrence displayed a longstanding peak during 18-45 months (HR >0.015), while hazard rates of extrathoracic recurrences were fairly low. In stage II (Figure 3B), the hazard rate of intra-thoracic recurrences was higher than stage I with a distinct peak during 15-24 months (HR $>0.04$ ). Flat peaks during 12-36 months were observed in the hazard rate curves of brain recurrence $(\mathrm{HR}=0.02)$ and bone recurrences $(\mathrm{HR}=0.01)$, respectively. There was a sharp peak $(\mathrm{HR}>0.07)$ during 12-21 months of intrathoracic recurrence in stage IIIA (Figure 3C). While brain and bone recurrences peaked during 6-18 months (HR $=0.03)$ and $15-27$ months $(\mathrm{HR}=0.02)$, respectively.

As histology was an independent predictor of both intra- and extra-thoracic recurrences based on MVA, we constructed hazard rate curves for brain, bone, abdomen, and chest according to the histology type (Figure 4). With respect to squamous cell carcinoma (Figure $4 A$ ), the hazard rates of bone and abdomen were low, with several small surges. There was a moderate peak $(H R>0.007)$ in brain recurrences at 9-15 months after surgery. The hazard rate of the chest was much higher than other regions and a sharp peak $(H R=0.023)$ occurred $10-18$ months after surgery. With respect to adenocarcinomas (Figure $4 B$ ), the hazard rate of chest recurrences showed the highest risk, followed by brain, bone, and abdomen. A long period of a high hazard rate (HR >0.013) at 12-33 months was noted in the curve of chest recurrences after surgery. The hazard rate of brain recurrences was high at 9-27 months $(\mathrm{HR}>0.007)$ with a peak of 0.008 . Notably, unlike the low, flat curve of bone recurrences in squamous cell carcinomas, the hazard rate curve of bone recurrences in adenocarcinomas displayed clear peaks at 15-30 months (HR >0.005). The mean specific DFS of bone metastases in adenocarcinomas was 105.4 months compared with 107.7 months in squamous cell carcinomas (HR: 0.642; 95\% CI: 0.413-0.998; $\mathrm{P}=0.047)$.

\section{Discussion}

Using population-based cancer surveillance data, we examined spatial-temporal recurrence dynamics in patients with NSCLC from stage I-IIIA who underwent curative surgical resection. Hazard function was used to visually reveal the transition of recurrence probabilities over time. We found that recurrences clustered at certain times after surgery, rather than irregularly.

In this study we reported that recurrences developed in $34.3 \%$ of the patients. Despite a high recurrence risk in NSCLC patients after surgery, the paucity of evidence precludes firm evidence-based recommendations about the best follow-up strategy for post-operative LC patients. The data mentioned above underline the importance of two aspects of follow-up regimens (i.e., when and where to perform the examinations). There are studies that have shown no difference in survival according to routine followup protocols compared to a symptom-based follow-up schedule (7), which were contradictory to the results of Choi et al. (8) and Williams et al. (9). The phase III IFCT0302 trial showed no difference between application of a chest X-ray (CXR) and CXR with CT in the detection of recurrences during follow-up (10). In spite of the divergence on surveillance, in the era of LC screening the incidence of early LC with favorable prognosis, small-sized tumors, and elderly patients has increased. Thus, intuitively it should implicate a closer and more structured follow-up strategy 


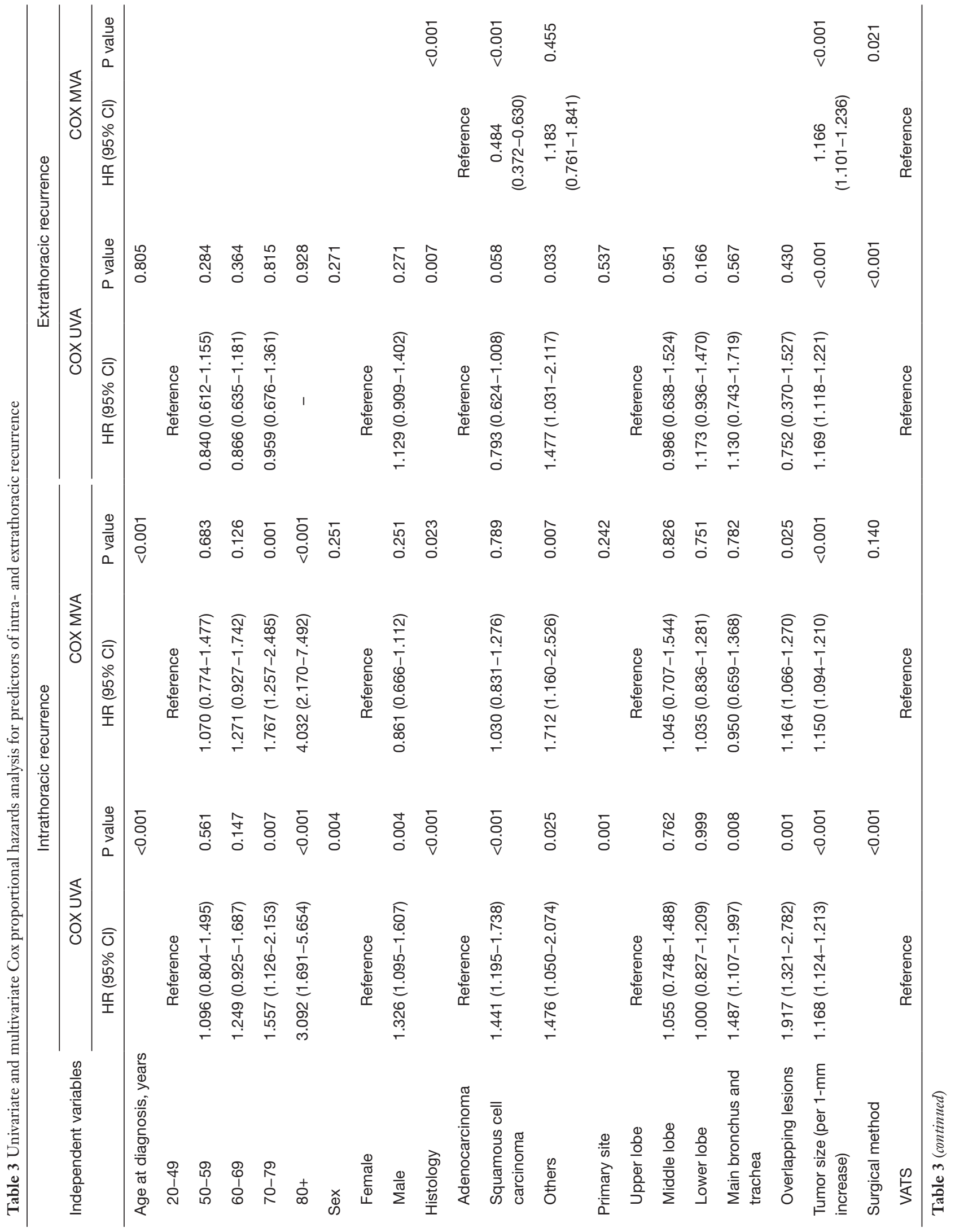




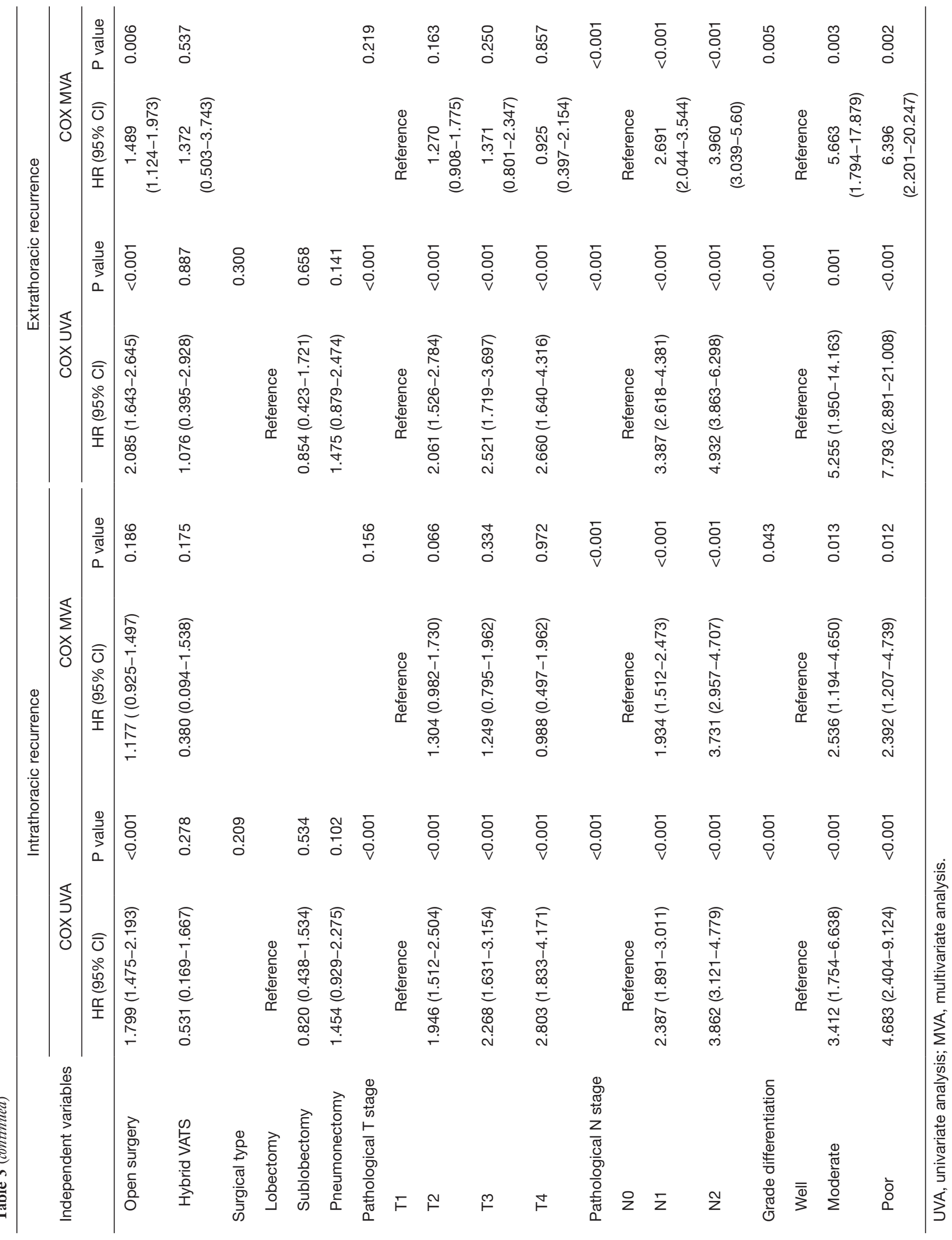




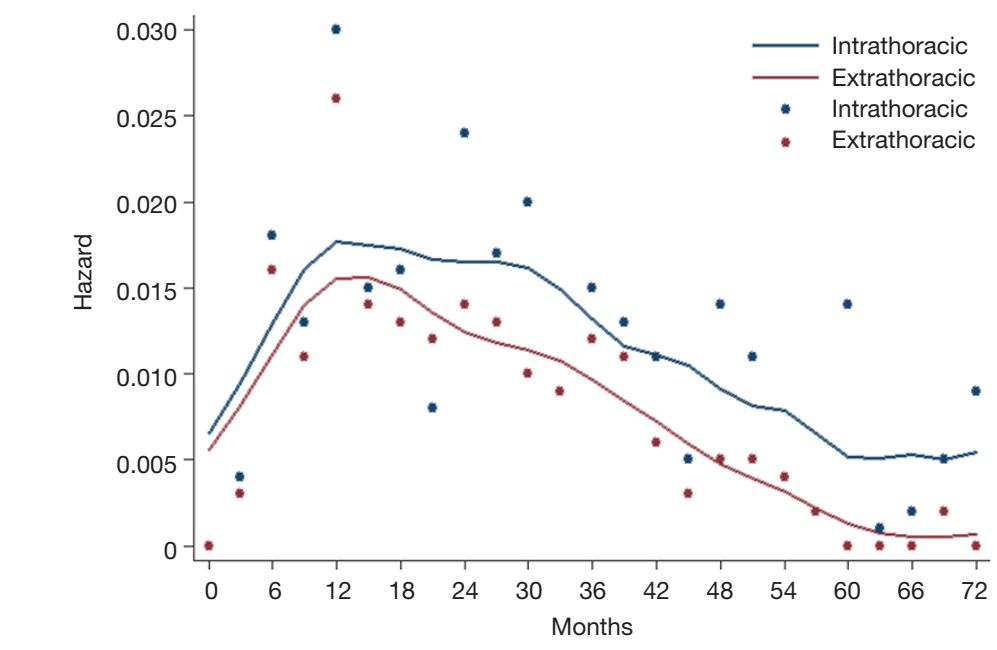

Number at risk $2486246523212051190116821564134611761000 \quad 836686575$

Figure 2 Hazard rate estimates for first event of intrathoracic and extrathoracic recurrences.

A

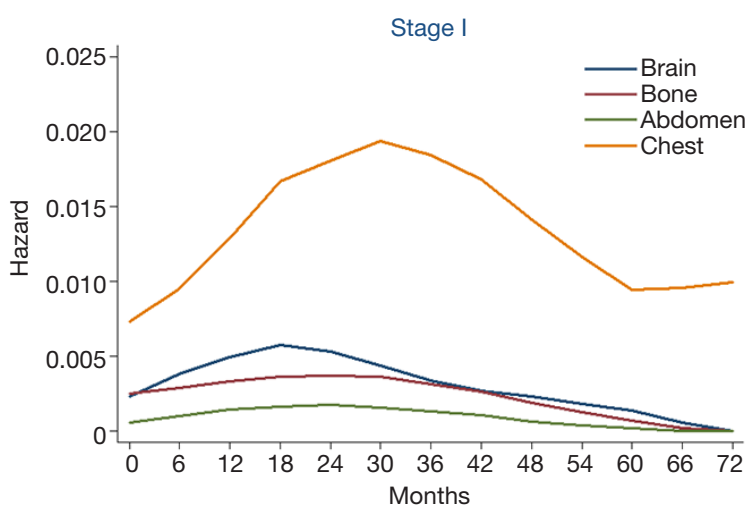

B

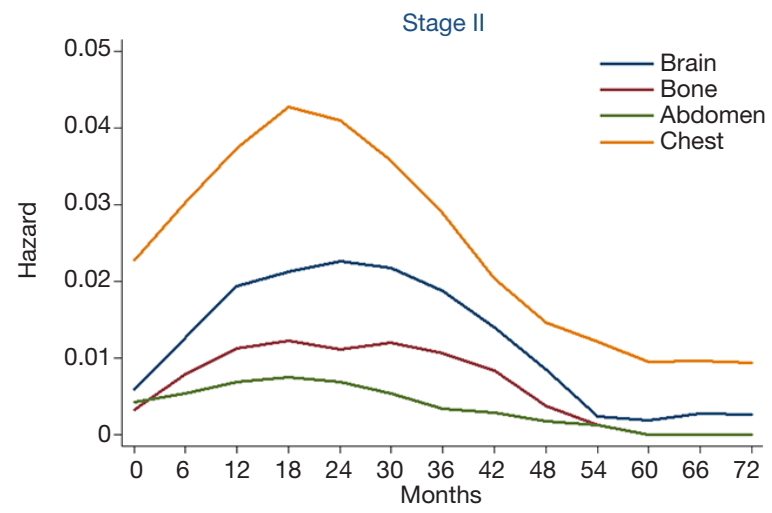

Number at risk $129812941268118711361047999 \quad 866 \quad 749641 \quad 544 \quad 437 \quad 366$ Number at risk $626 \quad 625 \quad 581 \quad 502 \quad 452 \quad 382 \quad 339288249211 \quad 183 \quad 153 \quad 131$

C

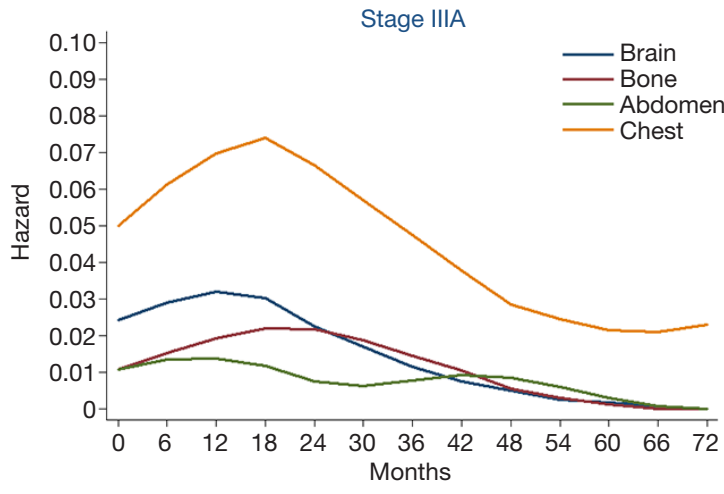

Number at risk $\begin{array}{lllllllllllll}562 & 546 & 472 & 362 & 313 & 253 & 226 & 192 & 178 & 148 & 109 & 91 & 78\end{array}$

Figure 3 Hazard rate according to stage. (A) Hazard rate estimates for first event of stage I; (B) hazard rate estimates for first event of stage II; (C) hazard rate estimated for first event of stage IIIA. 
A

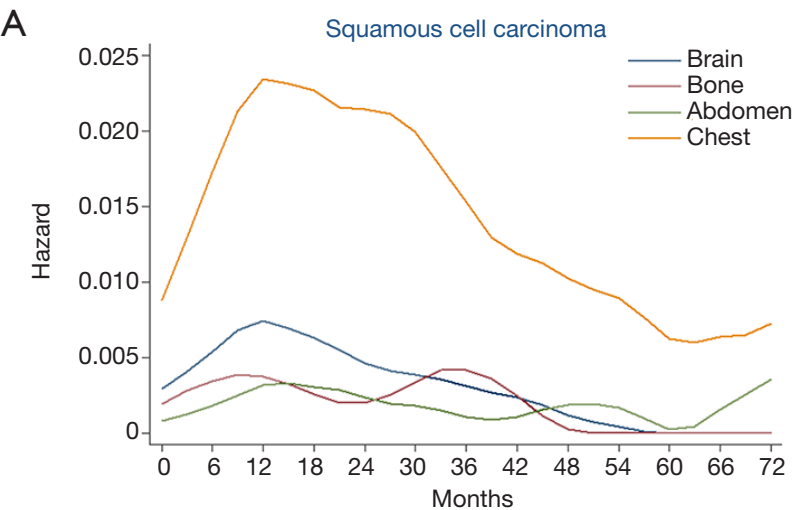

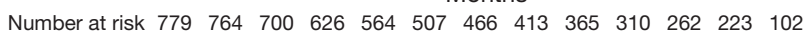

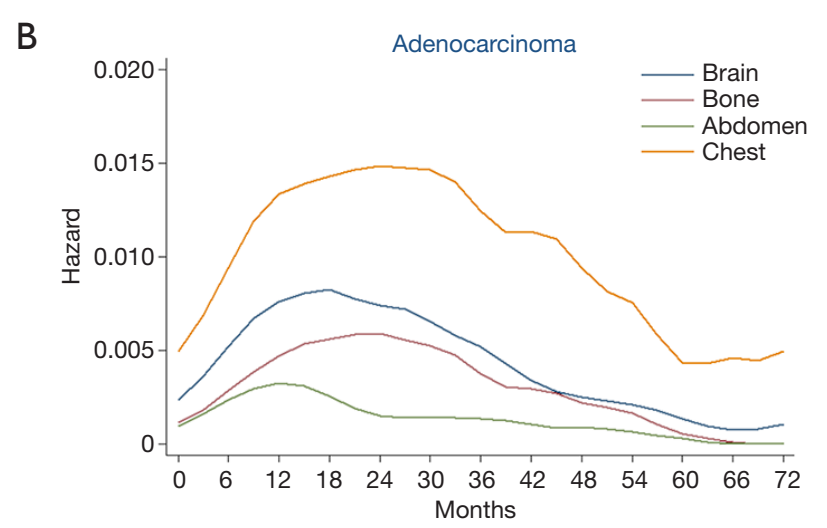

Number at risk $154115191427127611701049977 \quad 811 \quad 704 \quad 588 \quad 481382 \quad 317$

Figure 4 Hazard rate according to histology. (A) Hazard rate estimates for first event of squamous cell carcinoma according to recurrent regions; (B) hazard rate estimates for first event of adenocarcinoma according to recurrent regions.

that gives a better chance to detect recurrences of cancer that are amenable to curative intervention.

It is compelling that $78.5 \%$ of recurrences occurred in the first 3 years and greater than one-half of the recurrences occurred in the first 2 years after surgery. The initial peak of recurrence occurred approximately 12 months for both intra- and extra-thoracic recurrences. Luo et al. (11) reported that in 136 stage I lung adenocarcinoma patients, the post-recurrence survival for patients diagnosed with tumor recurrences shorter than 12 months after surgery was significantly worse (HR: 2.34; 95\% CI: 1.13-5.08, $\mathrm{P}=0.022$ ). Another study reported the cut-off point was 16 months, which suggests that early recurrence may refer to poor tumor biological behavior. This puts an emphasis on the surveillance to detect recurrences or second primary LCs in a timely manner within a short period of time after surgery.

A further interesting result was that intra-thoracic recurrences were prone to occur in squamous cell carcinomas, while extra-thoracic recurrences were prone to occur in adenocarcinomas. Tumor histology is known to be associated with local recurrences and distant metastases (12). Yamauchi et al. (13) studied NSCLC recurrences in 1,374 patients after complete resection and systemic lymph node dissection, a comparison of histology showed that the hazard rate of distant recurrence was $>3$ times higher in pN1-2 adenocarcinoma patients compared to the hazard rate of local recurrences. Squamous cell carcinoma patients had a negligible difference in risk. Clearly, adenocarcinomas have unique characteristics. Jensen et al. (14) reported that adenocarcinomas are more likely than squamous cell carcinomas to metastasize to the brain (18\% vs.9\%) and any of the major sites, including bone, brain, liver, adrenal, and lung (41\% vs. 27\%). Explanations may arise from adenocarcinomas showing stronger vascular invasion ability than squamous cell carcinomas. Differences in gene expression between these two histology types may also account for different recurrence patterns.

There is no agreement concerning follow-up regimens in guidelines, and current recommendations are not adjusted to histologic findings. The European Society of Medical Oncology (ESMO) guidelines recommend surveillance every 6 months for 2 years, including a chest CT at 12 and 24 months, and thereafter an annual visit, including history, physical examination, and chest CT scan [III, B] (15). According to the National Comprehensive Cancer Network (NCCN) guidelines, surveillance after completion of definitive therapy is adjusted to the stage; the follow-up for stage I-II is similar to the ESMO guidelines. With respect to stage III, more frequent surveillance is recommended, but no strict follow-up schedule is mentioned (16).

Based on the current situation and our results, there is no doubt that history taking and physical examinations should be performed regularly to assist in the evaluation of the patient's condition and tumor status. In our study, both intra-and extra-thoracic recurrences increased with stage. CT scans of appropriate frequency should be performed according to NCCN or ESMO guidelines to detect early intra-thoracic recurrences. With respect to extra-thoracic recurrences, individual surveillance could be adjusted to histologic findings and stage. A moderate hazard rate peak of brain was noted at 9-15 months in squamous cell carcinomas. Surveillance after curative 
resection is only useful if detection of recurrences results in a preferable prognosis or curative therapy. Curative treatment is often not suitable for all patients with extrathoracic recurrences but does work in select patients. With respect to oligo-metastases to the brain, radiosurgery or surgical removal are preferred alternatives, and are known to prolong survival (17). The targeted drug, alectinib, showed unequivocal efficacy in the phase III ALEX trial in reducing the incidence of CNS metastases and increasing the response rate (18). Therefore, early detection of brain metastases may afford patients an extended survival time and a practical recurrence pattern may result in rational drug use. While bone metastases are often not diagnosed in NSCLC patients until pain occurs and skeletal-related events (SREs), but if the first SRE occurs, subsequent events are going to follow, leading to a reduction in the quality of life. Thus, intensive surveillance at appropriate time for high-risk brain and bone recurrences should not be ignored. In our study, brain and bone showed high recurrence rates in stage II-IIIA. And brain recurrence in stage IIIA (6-18 months) occurred almost half the time of that in stage II (12-36 months), which requires an earlier and more intense surveillance. As for histology, there was a hazard rate peak in the brain at 9-27 months and a hazard rate peak in bone at 15-30 months for adenocarcinomas. A moderate peak of brain recurrence during 9-15 months was noted in squamous cell carcinoma. Thus, for stage IIIIIA patients, we recommend a brain MRI at 9-15 months for patients with both adenocarcinomas and squamous cell carcinomas, extra brain MRI at 15-27 months and emission computed tomography (ECT) scan of bone 15-30 months after surgery in patients with adenocarcinomas, especially those with larger tumors, lymph node metastases, open surgery, and poor differentiation. Detailed history taking and physical examination are clearly necessary; however, it is always important to remember that the desire of patients is a priority in developing a follow-up strategy, as brain MRI and bone ECT in detecting recurrences has not been routinely indicated. Survival benefit and cost should be taken into account.

This is the largest retrospective analysis about spatialtemporal recurrence patterns among patients with stage IIIIA NSCLC who underwent curative surgical resection. Despite several strengths, we recognize that our study had limitations. First, the study may contain several biases because of the retrospective approach, such as length of time bias and recall bias. Second, secondary primary LCs were excluded in our study. Surapaneni et al. (19) reported that the risk of developing a second primary LCs in stage I patients was highest in the first year and remained relatively lower, but constant until 10 years. This may have influenced our results to a small degree, but not lead to the contradiction because of a similar recurrence peak and low incidence. Finally, although the hazard rate was measured according to follow-up frequency at 3-month intervals, adherence to recommended surveillance should be taken into consideration. Further evidence, such as a prospective observational study, if not randomized controlled, is required to establish an accurate follow-up schedule in the future.

\section{Conclusions}

Based on our review of patients who underwent postoperative surveillance, intra- and extra-thoracic recurrences correlate with different clinicopathological factors. Brain MRI and bone ECT were recommended for selected patients in particular time to early detect extra-thoracic recurrences.

\section{Acknowledgements}

Our article has been language polished by International Science Editing Service.

Funding: This research received funding support from Outstanding Youth Foundation of Zhongshan Hospital (Project number: 2017ZSYQ28). The funding agency played no part in study design, data analysis, interpretation of data or manuscript preparation.

\section{Footnote}

Conflicts of Interest: The authors have no conflicts of interest to declare.

Ethical Statement: This study was approved by the Institutional Review Board of Shanghai Fudan University The informed consent of the biological and follow-up information being used in scientific research was obtained by preoperative signature for each patient.

\section{References}

1. Chen $W$, Zheng R, Baade PD, et al. Cancer statistics in China, 2015. CA Cancer J Clin 2016;66:115-32.

2. Goldstraw P, Chansky K, Crowley J, et al. The IASLC 
Lung Cancer Staging Project: Proposals for Revision of the TNM Stage Groupings in the Forthcoming (Eighth) Edition of the TNM Classification for Lung Cancer. J Thorac Oncol 2016;11:39-51.

3. Kelsey CR, Marks LB, Hollis D, et al. Local recurrence after surgery for early stage lung cancer: an 11-year experience with 975 patients. Cancer 2009;115:5218-27.

4. Virgo KS, McKirgan LW, Caputo MC, et al. Posttreatment management options for patients with lung cancer. Ann Surg 1995;222:700-10.

5. Younes RN, Gross JL, Deheinzelin D. Follow-up in lung cancer: how often and for what purpose? Chest 1999;115:1494-9.

6. Nishio M, Nakagawa K, Mitsudomi T, et al. Analysis of central nervous system efficacy in the J-ALEX study of alectinib versus crizotinib in ALK-positive non-small-cell lung cancer. Lung Cancer 2018;121:37-40.

7. Schmidt-Hansen M, Baldwin DR, Hasler E. What is the most effective follow-up model for lung cancer patients? A systematic review. J Thorac Oncol 2012;7:821-4.

8. Choi PJ, Jeong SS, Yoon SS. Prediction and prognostic factors of post-recurrence survival in recurred patients with early-stage NSCLC who underwent complete resection. J Thorac Dis 2016;8:152-60.

9. Williams BA, Sugimura H, Endo C, et al. Predicting postrecurrence survival among completely resected nonsmall-cell lung cancer patients. Ann Thorac Surg 2006;81:1021-7.

10. Westeel V, Lebitasy MP, Mercier M, et al. IFCT-0302 trial: randomised study comparing two follow-up schedules in completely resected non-small cell lung cancer. Rev Mal Respir 2007;24:645-52.

11. Luo J, Wang R, Han B, et al. Solid predominant histologic subtype and early recurrence predict poor postrecurrence survival in patients with stage I lung adenocarcinoma. Oncotarget 2017;8:7050-8.

12. Doebele RC, Lu X, Sumey C, et al. Oncogene status predicts patterns of metastatic spread in treatment-naive nonsmall cell lung cancer. Cancer 2012;118:4502-11.

13. Yamauchi Y, Muley T, Safi S, et al. The dynamic pattern of recurrence in curatively resected non-small cell lung cancer patients: Experiences at a single institution. Lung Cancer 2015;90:224-9.

14. Jensen GL, Tang C, Hess KR, et al. Patterns of metastatic progression after definitive radiation therapy for earlystage and locally advanced non-small cell lung cancer. Clin Exp Metastasis 2017;34:315-22.

15. Postmus PE, Kerr KM, Oudkerk M, et al. Early and locally advanced non-small-cell lung cancer (NSCLC): ESMO Clinical Practice Guidelines for diagnosis, treatment and follow-up. Ann Oncol 2017;28:iv1-iv21.

16. Ettinger DS, Wood DE, Aisner DL, et al. Non-Small Cell Lung Cancer, Version 5.2017, NCCN Clinical Practice Guidelines in Oncology. J Natl Compr Canc Netw 2017;15:504-35.

17. Sheehan JP, Sun MH, Kondziolka D, et al. Radiosurgery for non-small cell lung carcinoma metastatic to the brain: long-term outcomes and prognostic factors influencing patient survival time and local tumor control. J Neurosurg 2002;97:1276-81.

18. Peters S, Camidge DR, Shaw AT, et al. Alectinib versus Crizotinib in Untreated ALK-Positive Non-Small-Cell Lung Cancer. N Engl J Med 2017;377:829-38.

19. Surapaneni R, Singh P, Rajagopalan K, et al. Stage I lung cancer survivorship: risk of second malignancies and need for individualized care plan. J Thorac Oncol 2012;7:1252-6.
Cite this article as: Xi JJ, Yin JC, Wang L, Lu CL, Wang Q, Jiang W. A surveillance method-oriented detection of postoperative spatial-temporal recurrence for non-small cell lung cancer. J Thorac Dis 2018;10(11):6107-6117. doi: 10.21037/ jtd.2018.10.32 